\section{Unlocking thalidomide}

\author{
By Kai-Jye Lou, Staff Writer
}

Thalidomide has been used in humans since the 1950s, but the molecular mechanisms responsible for its known therapeutic and teratogenic effects are not well characterized. Researchers in Japan have now provided insight into the latter by showing that the developmental defects caused by thalidomide stem from the drug's ability to bind to cereblon. ${ }^{1}$ The findings provide the first step toward a blueprint for developing new thalidomide analogs and derivatives with reduced risk of birth defects.

The Japanese group used bead-based affinity purification studies to show that thalidomide binds to cereblon (CRBN), whereas a nonteratogenic analog, phthalimide, does not. CRBN is a component of an E3 ubiquitin ligase complex, which attaches ubiquitin molecules to their substrates to mark them for degradation.

Subsequent studies with a mutant CRBN that had very low thalidomide-binding activity indicated that thalidomide inhibits the E3 function of the CRBN-containing complex.

The finding suggests that thalidomide's inhibitory activity against the CRBN-containing complex promotes the accumulation of unknown substrates that lead to aberrant signaling in downstream developmental pathways (see Figure 1, "Molecular pathways involved in thalidomide teratogenicity").

"The affinity bead technology used in our study allowed us to identify CRBN as a target of thalidomide teratogenicity," said Hiroshi Handa, a professor in the Department of Biological Information at the Tokyo Institute of Technology. "We then used gene delivery methods in zebrafish and chicks to show that thalidomide teratogenicity could be prevented."

Thalidomide is known to be teratogenic in primates, rabbits and chicks, but not in mice and rats.

In a new zebrafish model, researchers showed that embryos overexpressing wild-type crbn (zcrbn) were sensitive to thalidomide-induced developmental defects. In contrast, embryos that overexpressed a mutant zcrbn with very low thalidomide binding were not sensitive to defects.

The researchers repeated the study in chicks and showed that overexpression of mutant human CRBN reduced the severity of thalidomide-induced forelimb malformations compared with overexpression of wild-type human CRBN.

"Thalidomide has diverse effects, which include sedation, therapeutic efficacy against leprosy and multiple myeloma and the teratogenic side effects," said Handa, corresponding author on the Science paper. "The identification of CRBN as a thalidomide-binding protein is a

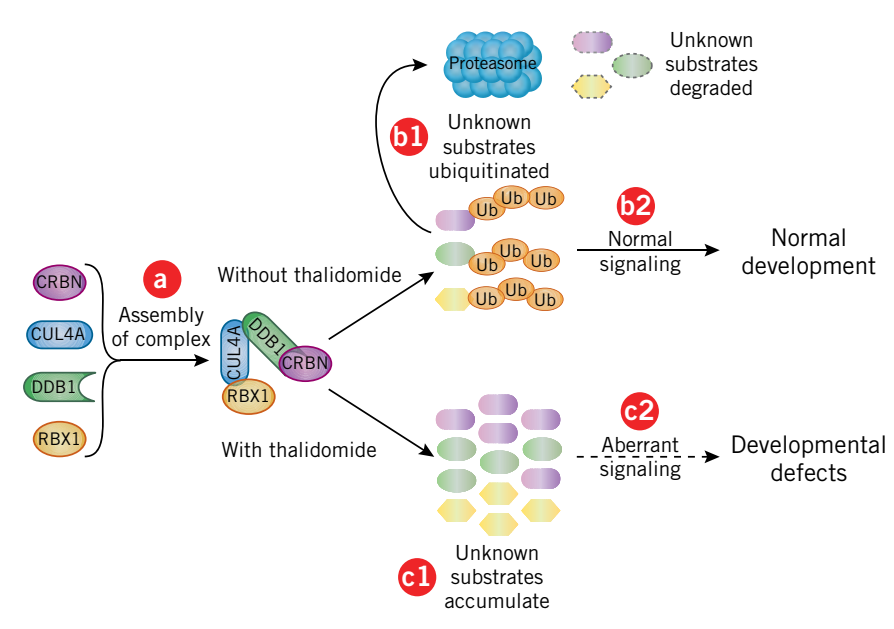

Figure 1. Molecular pathways involved in thalidomide teratogenicity. Although thalidomide is known to cause developmental defects in both humans and animal models, the molecular mechanisms responsible for its teratogenicity were not known. In a paper in Science, researchers showed that the teratogenic effects of thalidomide stem from the drug binding to cereblon (CRBN), a component in an E3 ubiquitin ligase complex.

CRBN assembles with damage-specific DNA-binding protein 1 (DDB1), cullin 4A (CUL4A) and ring-box 1 (RBX1; ROC1) to form an E3 ubiquitin ligase complex [a].

Under normal conditions, this E3 complex ubiquitinates its substrates, allowing subsequent proteasome degradation [b(1)]. Signaling in downstream pathways is preserved and developmental processes proceed as normal [b(2)].

When thalidomide is present, it binds to CRBN and inhibits the E3 ligase function of the complex. As a result, the substrates are not ubiquitinated, leading to their aberrant accumulation [c(1)]. This causes irregular signaling in downstream pathways, leading to the classic developmental defects associated with thalidomide, such as malformed limbs and ears [c(2)].

The substrates of the CRBN-containing E3 complex relevant to thalidomide teratogenicity are not yet known. However, the researchers showed that CRBN with a mutated thalidomide-binding site can prevent thalidomide-induced repression of fibroblast growth factor 8 (FGF8), a key regulator of development.

Thus, the researchers suggested that an inhibitor of FGF8 production could be a downstream target of thalidomide and the CRBN-containing E3 complex.

significant step towards the development of new drugs that don't have such side effects."

\section{Investing in derivatives}

Handa told SciBX that additional insights on thalidomide's molecular mechanisms are still needed before the findings can be applied to drug 


\section{ANALYSIS}

development. The issue, he said, is that "we don't know the real targets that are responsible for thalidomide's therapeutic effects. If CRBN is a common target to both therapeutic effects and teratogenic effects, it needs to be elucidated how the beneficial effects and teratogenic effects are transmitted through CRBN to downstream signaling pathways. Understanding of the different pathways will lead to new drug development."

Teasing out these molecular targets could therefore facilitate the development of analogs and derivatives with improved potency and could help screen out compounds with teratogenic risk at an early stage of drug development.

Thalidomide originally was introduced as a sedative in Europe in the 1950s but was withdrawn from the market after it was linked to birth defects. Celgene Corp. has since reintroduced the drug as Thalomid to treat leprosy and multiple myeloma (MM). Its use is strictly regulated under a risk management program.

The company also markets Revlimid lenalidomide, a thalidomide analog, to treat MM and myelodysplastic syndrome (MDS). The labels for both drugs contain black box warnings on potential teratogenicity. In 2009, sales of Revlimid and Thalomid were \$1.7 billion and $\$ 437$ million, respectively.

Celgene had developed Revlimid with the goal of creating a nonteratogenic version of thalidomide and initially reported no evidence of teratogenicity in preclinical and clinical trials. ${ }^{2}$ Although Revlimid's original 2005 label contained a black box warning on teratogenicity, the concern was based primarily on the drug's structural similarity to thalidomide.

\section{TARGETS \& MECHANISMS}

A revised Revlimid label in February 2009 included experimental evidence of teratogenicity and noted that "lenalidomide produced malformations in the offspring of female monkeys who received the drug during pregnancy, similar to birth defects observed in humans following exposure to thalidomide during pregnancy."

Handa said his group is now isolating and identifying additional thalidomide targets to understand the correlations between CRBN and the drug's therapeutic effects. "We are also trying to identify substrates that are ubiquitinated by the CRBN-containing E3 ubiquitin ligase to understand downstream signals of CRBN," he added.

Handa said the Tokyo Institute of Technology has filed a patent application covering screening for compounds with activity against CRBN. The work is available for licensing from the institute's Office of Industry Liaison.

Celgene declined to comment on the Science paper.

Lou, K.-J. SciBX 3(13); doi:10.1038/scibx.2010.396

Published online April 1, 2010

\section{REFERENCES}

1. Ito, T. et al. Science; published online March 11, 2010; doi:10.1126/science. 1177319

Contact: Hiroshi Handa, Tokyo Institute of Technology, Tokyo, Japan e-mail: handa.h.aa@m.titech.ac.jp

Contact: Takumi Ito, same affiliation as above e-mail: ito.t.aj@m.titech.ac.jp

2. Flanagan, M. BioCentury 13(45), A6; Oct. 10, 2005

COMPANIES AND INSTITUTIONS MENTIONED

Celgene Corp. (NASDAQ:CELG), Summit, N.J.

Tokyo Institute of Technology, Tokyo, Japan 